\title{
Multiinstitutional prospective observational study of stereotactic radiosurgery for patients with multiple brain metastases from non-small cell lung cancer (JLGK0901 study-NSCLC)
}

\author{
Takashi Shuto, MD, ${ }^{1}$ Atsuya Akabane, MD, ${ }^{2}$ Masaaki Yamamoto, MD, ${ }^{3}$ Toru Serizawa, MD, ${ }^{4}$ \\ Yoshinori Higuchi, MD, ${ }^{5}$ Yasunori Sato, PhD, ${ }^{6}$ Jun Kawagishi, MD, ${ }^{7}$ Kazuhiro Yamanaka, MD, ${ }^{8}$ \\ Hidefumi Jokura, MD, ${ }^{7}$ Shoji Yomo, MD, ${ }^{9}$ Osamu Nagano, MD, ${ }^{10}$ and Hidefumi Aoyama, MD ${ }^{11}$ \\ 'Department of Neurosurgery, Yokohama Rosai Hospital, Yokohama, Kanagawa; ${ }^{2}$ Gamma Knife Center, NTT Medical Center \\ Tokyo; ${ }^{3}$ Katsuta Hospital Mito Gamma House, Hitachinaka, Ibaraki; ${ }^{T}$ Tokyo Gamma Unit Center, Tsukiji Neurological Clinic, \\ Tokyo; ${ }^{5}$ Neurological Surgery, Chiba University Graduate School of Medicine, Chiba; ${ }^{6}$ Department of Preventive Medicine and \\ Public Health, Keio University School of Medicine, Tokyo; ' Jiro Suzuki Memorial Gamma House, Furukawa Seiryo Hospital, \\ Sendai, Miyagi; ${ }^{8}$ Department of Neurosurgery, Osaka City University Graduate School of Medicine, Osaka; ${ }^{9}$ Saitama Gamma \\ Knife Center, Sanai Hospital, Saitama; ${ }^{10} \mathrm{Gamma}$ Knife House, Chiba Cerebral and Cardiovascular Center, Ichihara, Chiba; and \\ ${ }^{11}$ Department of Radiology, Niigata University Graduate School of Medicine and Dental Sciences, Niigata, Japan
}

OBJECTIVE Previous Japanese Leksell Gamma Knife Society studies (JLGK0901) demonstrated the noninferiority of stereotactic radiosurgery (SRS) alone as the initial treatment for patients with 5-10 brain metastases (BMs) compared with those with 2-4 BMs in terms of overall survival and most secondary endpoints. The authors studied the aforementioned treatment outcomes in a subset of patients with BMs from non-small cell lung cancer (NSCLC).

METHODS Patients with initially diagnosed BMs treated with SRS alone were enrolled in this prospective observational study. Major inclusion criteria were the existence of up to 10 tumors with a maximum diameter of less than $3 \mathrm{~cm}$ each, a cumulative tumor volume of less than $15 \mathrm{~cm}^{3}$, and no leptomeningeal dissemination in patients with a Karnofsky Performance Scale score of $70 \%$ or better.

RESULTS Among 1194 eligible patients, 784 with NSCLC were categorized into 3 groups: group A (1 tumor, $n=299$ ), group $B(2-4$ tumors, $n=342)$, and group $C(5-10$ tumors, $n=143)$. The median survival times were 13.9 months in group A, 12.3 months in group $B$, and 12.8 months in group $C$. The survival curves of groups $B$ and $C$ were very similar (hazard ratio [HR] 1.037; 95\% Cl 0.842-1.277; $p<0.0001$, noninferiority test). The crude and cumulative incidence rates of neurological death, deterioration of neurological function, newly appearing lesions, and leptomeningeal dissemination did not differ significantly between groups B and C. SRS-induced complications occurred in $145(12.1 \%)$ patients during the median post-SRS period of 9.3 months (IQR 4.1-17.4 months), including 46, 54, 29, 11, and 5 patients with a Common Terminology Criteria for Adverse Events v3.0 grade 1, 2, 3, 4, or 5 complication, respectively. The cumulative incidence rates of adverse effects in groups $A, B$, and C 60 months after SRS were $13.5 \%, 10.0 \%$, and $12.6 \%$, respectively (group B vs C: HR 1.344; 95\% Cl 0.768-2.352; $p=0.299$ ). The 60 -month post-SRS rates of neurocognitive function preservation were $85.7 \%$ or higher, and no significant differences among the 3 groups were found.

CONCLUSIONS In this subset analysis of patients with NSCLC, the noninferiority of SRS alone for the treatment of 5-10 versus 2-4 BMs was confirmed again in terms of overall survival and secondary endpoints. In particular, the incidence of neither post-SRS complications nor neurocognitive function preservation differed significantly between groups

ABBREVIATIONS BM = brain metastasis; CTCAE = Common Terminology Criteria for Adverse Events v3.0; EGFR = epidermal growth factor receptor; HR = hazard ratio; HVLT-R = Hopkins Verbal Learning Test-Revised; IQR = interquartile range; JLGK = Japanese Leksell Gamma Knife; KPS = Karnofsky Performance Scale; MMSE = Mini-Mental State Examination; MST = median survival time; NCF = neurocognitive function; NSCLC = non-small cell lung cancer; SRS = stereotactic radiosurgery; TKI = tyrosine kinase inhibitor; WBRT = whole-brain radiotherapy.

SUBMITTED May 30, 2018. ACCEPTED July 19, 2018.

INCLUDE WHEN CITING DOI: 10.3171/2018.7.GKS181378. 
$B$ and $C$. These findings further strengthen the already-reported noninferiority hypothesis of SRS alone for the treatment of patients with $5-10 \mathrm{BMs}$.

https://thejns.org/doi/abs/10.3171/2018.7.GKS181378

KEYWORDS stereotactic radiosurgery; Gamma Knife; non-small cell lung cancer; complications; neurocognitive function; oncology

$\mathrm{O}$ UR previous multiinstitutional prospective observational study (a clinical trial conducted by the Japanese Leksell Gamma Knife Society [JLGK0901]) assessed 1194 patients with brain metastases (BMs) initially managed with stereotactic radiosurgery (SRS) using the Gamma Knife (Elekta) without whole-brain radiotherapy (WBRT). ${ }^{23}$ A comparison of median survival times (MSTs) in 2 patient groups (i.e., 10.8 months in patients with 5-10 tumors and 10.8 months in those with 2-4 tumors) revealed no difference in outcomes (hazard ratio [HR] 0.974; 95\% CI 0.806-1.177 [below the noninferiority margin]; $\mathrm{p}=0.78 ; \mathrm{p}<0.0001$, noninferiority test). Furthermore, we found no significant differences in the crude or cumulative incidence of neurological death, deterioration of neurological function, local recurrence, new lesion appearance, leukoencephalopathy, rates of salvage SRS, or rates of WBRT between the 2 groups based on the number of BMs. However, the median observational time in the study (10.7 months; interquartile range [IQR] 5.818.8 months) was not sufficient to confirm the long-term safety of Gamma Knife SRS treatment alone for 5-10 BMs. Therefore, we extended the observation period for 2 more years (up to 2014) with the main aim of examining neurocognitive function (NCF) preservation. We used the Mini-Mental State Examination (MMSE) test and late SRS-related complications to assess NCF. ${ }^{22}$

Using a heterogeneous cohort and including all primary cancer types, particularly all lung cancer subtypes, even small cell cancer, was a major weakness of our previous investigations. ${ }^{22,23}$ Therefore, in this study, we reappraised whether the aforementioned hypothesis would be applicable to a homogeneous subset of patients with only non-small cell lung cancer (NSCLC). We focused especially on NCF preservation and the development of delayed SRS-related complications. We also assessed the roles of systemic anticancer agents, particularly molecular targeting agents, in terms of post-SRS patient survival.

\section{Methods}

This prospective observational study involved $23 \mathrm{Gam}$ ma Knife facilities in Japan. Informed written consent was obtained from each patient before enrollment, and the institutional review board of each participating facility had already approved all aspects of the study. Before patient recruitment, the study was registered with the University Medical Information Network Clinical Trial Registry (no. 000001812; see http://www.umin.ac.jp/ctr/index.htm).

The JLGK0901 inclusion criteria applied at the time of SRS were as follows: 1) newly diagnosed BMs that had been confirmed by contrast-enhanced MRI within 6 weeks of SRS, 2) $\leq 10$ tumors, 3$)<10-\mathrm{cm}^{3} /<3.0-\mathrm{cm}$ maximum volume/diameter ratio of the largest tumor, 4) a cumulative tumor volume of $\leq 15.0 \mathrm{~cm}^{3}, 5$ ) no evidence of menin- geal dissemination, and 6) a Karnofsky Performance Scale $(\mathrm{KPS})^{11}$ score of $\geq 70 \%$ or, in patients with a KPS score of $<70 \%$, a reasonable expectation of neurological function improvement in response to SRS..$^{17,18,23}$ All original malignant tumor types except sarcoma and lymphoma were considered to be acceptable for treatment. Exclusion criteria were as follows: 1) 2 or more original malignant tumors, 2) patient pregnancy or breastfeeding, 3) previously diagnosed psychological disorder(s), 4) contraindications for MRI examination or use of a gadolinium agent, and 5) any previous surgery and/or irradiation to the skull or brain.

The study protocol (i.e., the SRS techniques used), our follow-up protocol, clinical outcome endpoints, and study management were described in our earlier reports ${ }^{17,18,23}$ and thus are not detailed herein. In brief, each patient was categorized into 1 of 3 groups, namely, group A (1 tumor), group B (2-4 tumors), or group C (5-10 tumors). Then, the noninferiority of treatment results for group $\mathrm{C}$ to that of group B was investigated based on the primary and secondary endpoints. The effects of targeted therapy were analyzed also. NCF was assessed using the MMSE scores obtained 4 and 12 months after SRS and at 12-month intervals thereafter. MMSE score preservation was defined as a score reduction of less than 3 from the baseline value. Complications caused by SRS were graded according to the Common Terminology Criteria for Adverse Events v3.0 (CTCAE).${ }^{16}$ For the secondary endpoints, although we endeavored to reanalyze crude and cumulative incidence rates of leukoencephalopathy, our study protocol did not include a grading system for it; thus, we were able to assess only the presence of leukoencephalopathy versus its absence. The criteria for the 2 major endpoints (i.e., complications and leukoencephalopathy) were given in detail in our previous reports. ${ }^{17,18,23}$

The details of our statistical analysis methods also were provided in previous reports ${ }^{22,23}$ and are summarized here.

For analyses of the primary endpoint, we applied the Cox proportional hazards model with prognostic factors as covariates. For analyses of the secondary endpoints, time-to-event outcomes, we used competing risk with the Fine-Gray generalization of the proportional hazards model, which accounts for death as a competing risk. ${ }^{8,10}$

We assessed MMSE scores by using 4 different methods, which was deemed necessary because many subsets of the MMSE lacked follow-up data. Details are provided in our previous report.22

All of the statistical analyses were carried out by a statistician (Y.S.), who was not involved in either SRS treatment or patient follow-up, using SAS 9.3 software (SAS Institute).

\section{Results}

We recruited 1194 patients eligible for this study between 
TABLE 1. Summary of patient characteristics

\begin{tabular}{|c|c|c|c|c|c|}
\hline Variable & Total $(n=784)$ & Group A $(n=299)$ & Group B $(n=342)$ & Group C $(n=143)$ & $p$ Value \\
\hline \multicolumn{6}{|l|}{ Age in yrs } \\
\hline Mean \pm SD & $66.1 \pm 9.8$ & $66.6 \pm 10$ & $65.4 \pm 9.9$ & $66.9 \pm 8.9$ & 0.13 \\
\hline Range & $34-91$ & $34-91$ & $37-89$ & $44-86$ & \\
\hline Age $\geq 65$ yrs (no. [\%]) & $466(59)$ & $185(62)$ & $196(57)$ & $85(59)$ & 0.50 \\
\hline Female sex (no. [\%]) & $275(35)$ & $107(36)$ & $113(33)$ & $55(38)$ & 0.49 \\
\hline KPS score $\geq 80$ (no. [\%]) & $696(89)$ & $259(87)$ & $307(90)$ & $130(91)$ & 0.30 \\
\hline \multicolumn{6}{|l|}{ Extracranial disease } \\
\hline Complete or partial response & $68(9)$ & $29(10)$ & $31(9)$ & $8(6)$ & 0.30 \\
\hline Stable disease & $456(58)$ & $182(61)$ & $190(56)$ & $84(59)$ & \\
\hline Progressive disease & $260(33)$ & $88(29)$ & $121(35)$ & $51(36)$ & \\
\hline \multicolumn{6}{|l|}{ RPA class } \\
\hline 1 & $209(27)$ & $80(27)$ & $93(27)$ & $36(25)$ & 0.52 \\
\hline 2 & $553(71)$ & 207 (69) & $241(70)$ & $105(73)$ & \\
\hline 3 & $22(3)$ & $12(4)$ & $8(2)$ & $2(1)$ & \\
\hline Total tumor volume in $\mathrm{cm}^{3}$ & & & & & $<0.0001$ \\
\hline Mean \pm SD & $2.41 \pm 2.7$ & $1.98 \pm 2.2$ & $2.56 \pm 2.8$ & $2.94 \pm 2.9$ & \\
\hline Range & $0.008-14.9$ & $0.008-9.9$ & $0.02-14.9$ & $0.024-13.9$ & \\
\hline Total tumor volume $\geq 1.9 \mathrm{~cm}^{3}$ (no. [\%]) & $338(43)$ & $112(37)$ & $150(44)$ & $76(53)$ & 0.007 \\
\hline Max largest tumor diameter in $\mathrm{cm}$ & & & & & 0.99 \\
\hline Mean \pm SD & $1.51 \pm 0.6$ & $1.51 \pm 0.6$ & $1.51 \pm 0.6$ & $1.50 \pm 0.6$ & \\
\hline Range & $0.08-2.99$ & $0.30-2.98$ & $0.11-2.99$ & $0.08-2.97$ & \\
\hline Max largest tumor diameter $\geq 1.6 \mathrm{~cm}(\mathrm{no} .[\%])$ & $337(43)$ & $129(43)$ & $146(43)$ & $62(43)$ & 0.98 \\
\hline MMSE score & & & & & 0.79 \\
\hline Median & 28 & 28 & 28 & 28 & \\
\hline Range & $7-30$ & $7-30$ & $17-30$ & $11-30$ & \\
\hline MMSE score $\geq 27$ (no. [\%]) & $520(66)$ & $190(64)$ & $232(68)$ & $98(69)$ & 0.41 \\
\hline \multicolumn{6}{|l|}{ Neurological symptoms } \\
\hline Yes (no. [\%]) & $198(25)$ & $75(25)$ & $87(25)$ & $36(25)$ & 0.99 \\
\hline
\end{tabular}

Max = maximum; RPA = recursive partitioning analysis.

The sums of percentages might not equal 100 due to rounding.

February 2009 and February 2012. Data acquisition was extended to the end of December 2014, and the database was then finalized at the end of December 2014. Among the 1194 patients, we studied 784 with NSCLC. Clinical characteristics of the patient population as a whole and according to the 3 tumor-number groups are summarized in Table 1 . We found no imbalances among the groups in regard to most of the clinical characteristics (i.e., age, sex, extracranial disease status, initial KPS score, Radiation Therapy Oncology Group recursive partitioning analysis class, neurological symptoms, and maximum diameter of the largest tumor). The only exception was total tumor volumes, which differed significantly among the 3 groups.

In total, 672 (85.7\%) patients died during the observational period. Among these deceased patients, 609 (90.6\%) died as a result of systemic disease (Table 2). Crude and cumulative incidence rates of neurological death, deterioration of neurological function, newly appearing lesions, and leptomeningeal dissemination did not differ significantly between groups B and C. The proportions of deaths attributed to intracranial lesions were similar in the 3 groups.

The post-SRS MST of group A (13.9 months; 95\% CI
11.7-17.6 months) was significantly longer than that of group B (12.3 months; 95\% CI 10.6-13.7 months) (HR 0.844 ; 95\% CI 0.712-0.999; $\mathrm{p}=0.048$ ) (Fig. 1), whereas the post-SRS MSTs of group C (12.8 months; $95 \%$ CI 10.8-16.0 months) and group B were very similar (HR $1.037 ; 95 \%$ CI 0.842-1.277; $p=0.73 ; p<0.0001$, noninferiority test). This subset evaluation confirmed the noninferiority of Gamma Knife SRS alone for managing patients with 5-10 tumors compared with those with 2-4 tumors. Multivariable analysis of clinical factors before SRS revealed that BM, female sex, age of $<65$ years, a KPS score of $\geq 80$, having no neurological deficits, and having stable extracranial lesions correlated significantly with a long survival period (Table 3 ).

Regarding the administration of systemic anticancer agents after SRS, we categorized each of our 784 patients into 1 of the following 4 groups: 1) a chemotherapeutic agent(s) alone (305 patients), 2) a molecular targeting agent(s) alone (78 patients), 3) both chemotherapeutic and molecular targeting agents (205 patients), or 4) none of these regimens (196 patients). Overall survival curves for these 4 groups are presented in Fig. 2. The post-SRS MST 
TABLE 2. Treatment outcomes after SRS

\begin{tabular}{lccccccc}
\hline & Total & Group A & Group B & Group C & \multicolumn{3}{c}{ Group B vs C } \\
\cline { 6 - 8 } & $(\mathrm{n}=784)$ & $(\mathrm{n}=299)$ & $(\mathrm{n}=342)$ & $(\mathrm{n}=143)$ & $\mathrm{HR}$ & $95 \% \mathrm{Cl}$ & $\mathrm{p} \mathrm{Value}$ \\
\hline Death & $672(85.7)$ & $251(83.9)$ & $294(86.0)$ & $127(88.8)$ & 1.037 & $0.842-1.277$ & 0.73 \\
\hline Neurological death & $63(8.0)$ & $30(10.0)$ & $20(5.8)$ & $13(9.1)$ & 1.583 & $0.789-3.179$ & 0.19 \\
\hline Deterioration of neurological function & $110(14.0)$ & $47(15.7)$ & $43(12.6)$ & $20(14.0)$ & 1.109 & $0.655-2.080$ & 0.707 \\
\hline New lesion appearance & $464(59.2)$ & $155(51.8)$ & $213(62.3)$ & $96(67.1)$ & 1.216 & $0.951-1.555$ & 0.118 \\
\hline Leptomeningeal dissemination & $124(15.8)$ & $45(15.1)$ & $49(14.3)$ & $30(21.0)$ & 1.555 & $0.989-2.452$ & 0.057 \\
\hline Leukoencephalopathy & $6(0.8)$ & $2(1.0)$ & $2(1.0)$ & $2(1.4)$ & 2.446 & $0.345-17.33$ & 0.370 \\
\hline Salvage SRS & $347(44.3)$ & $115(38.5)$ & $162(47.4)$ & $70(49.0)$ & 1.053 & $0.793-1.399$ & 0.719 \\
\hline Salvage WBRT & $84(10.7)$ & $26(8.7)$ & $39(11.4)$ & $19(13.3)$ & 1.119 & $0.650-1.929$ & 0.684 \\
\hline
\end{tabular}

Data are presented as the number (\%) of patients unless otherwise indicated.

in patients given a targeting agent(s) only (15.1 months; 95\% CI 12.3-24.4 months) was significantly longer than that in patients treated only with a chemotherapeutic agent(s) (11.0 months; 95\% CI 10.0-12.8 months) (HR $0.70 ; 95 \%$ CI $0.53-0.92 ; p=0.0112)$. The post-SRS MST in patients given both molecular targeting and chemotherapeutic agents (26.4 months; 95\% CI 21.7-30.5 months) was significantly longer than that in patients given only a chemotherapeutic agent(s) (HR 0.70; 95\% CI 0.53-0.92; p
$<0.0001)$. However, the MST of patients given both types of agents was not significantly longer than that in patients treated with a molecular targeting agent(s) only (HR 0.88; 95\% CI 0.76-1.02; $\mathrm{p}=0.101$ ).

SRS-related complications developed in 88 (11\%) patients during the median post-SRS follow-up period of 10.1 months (range 0.3-58.1 months; IQR 5.3-17.5 months). The cumulative incidence rates of SRS-related complications, determined by applying a competing risk

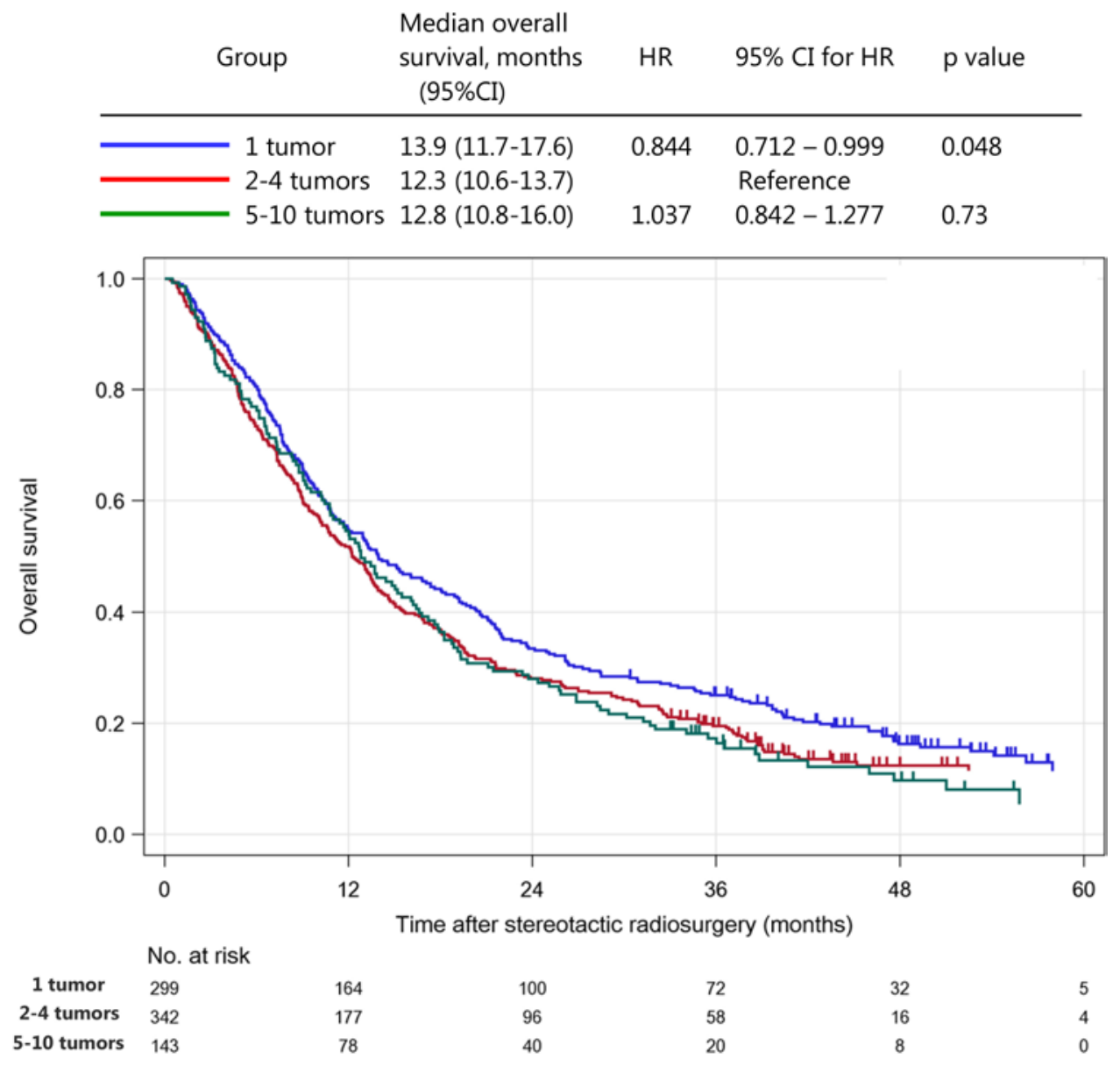

FIG. 1. Kaplan-Meier curves of overall survival of patients in the 3 groups in this study. 
TABLE 3. Clinical factors that affected survival after SRS

\begin{tabular}{lccc}
\hline \multicolumn{1}{c}{ Parameter } & HR & $95 \% \mathrm{Cl}$ & $\mathrm{p} \mathrm{Value}$ \\
\hline Group & & & \\
\hline A vs B & 0.841 & $0.709-0.999$ & 0.048 \\
\hline B vs C & 1.044 & $0.845-1.291$ & 0.687 \\
\hline Sex, male vs female & 1.440 & $1.224-1.694$ & $<0.0001$ \\
\hline Age, $\geq 65$ vs $<65$ yrs & 1.397 & $1.194-1.635$ & $<0.0001$ \\
\hline KPS score, $<80$ vs $\geq 80$ & 1.934 & $1.496-2.500$ & $<0.0001$ \\
\hline $\begin{array}{l}\text { Max diameter of largest tumor, } \geq 1.6 \\
\quad \text { vs }<1.6 \text { cm }\end{array}$ & 1.075 & $0.791-1.462$ & 0.644 \\
\hline Total tumor volume, $\geq 1.9$ vs $<1.9 \mathrm{~cm}^{3}$ & 1.083 & $0.796-1.474$ & 0.612 \\
\hline Neurological symptoms, yes vs no & 1.297 & $1.051-1.600$ & 0.015 \\
\hline $\begin{array}{l}\text { Extracranial disease, not controlled } \\
\text { vs controlled }\end{array}$ & 1.215 & $1.035-1.428$ & 0.017 \\
\hline
\end{tabular}

analysis to the 3 tumor-number groups, are shown in Fig. 3. At the 5-year follow-up point, the cumulative incidence rates were $13.5 \%, 10.0 \%$, and $12.6 \%$ in groups $\mathrm{A}, \mathrm{B}$, and $\mathrm{C}$, respectively. We detected no significant intergroup differences (group A vs B, HR 1.171, 95\% CI 0.731-1.876, p $=0.512$; group B vs C, HR 1.344, 95\% CI 0.768-2.352, p $=0.299$ ). Among the 88 patients who developed an SRSrelated complication, 26, 33, 20, 6, and 3 were categorized as having a CTCAE grade 1,2,3, 4, or 5 complication, respectively. ${ }^{16}$ The CTCAE grade distributions did not differ markedly between groups B and C $(p=0.3379)$. Among the pre-SRS clinical factors examined, multivariable analysis revealed that age ( $\geq 65$ vs $<65$ years) and neurological symptoms (yes vs no) correlated significantly with higher incidence rates of SRS-related complications (Table 4).

As already discussed, 78 patients received a molecular targeting agent(s) alone. Among these patients, 6 (7.7\%) developed an SRS-related complication. Neither univariable (HR 1.295; 95\% CI 0.849-1.974; $\mathrm{p}=0.230$ ) nor

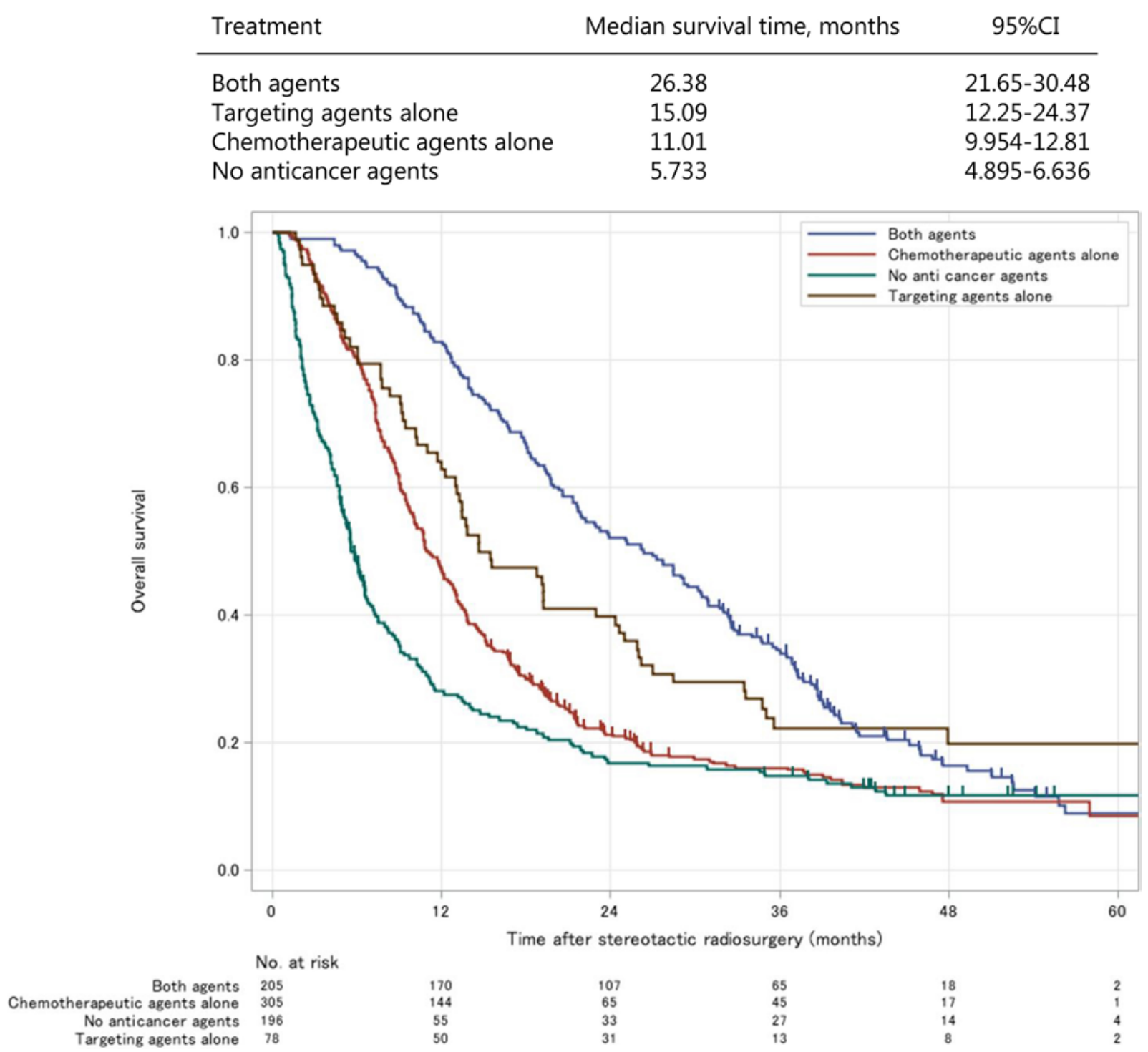

FIG. 2. Kaplan-Meier curves of overall survival in patients with or without an anticancer agent(s). 


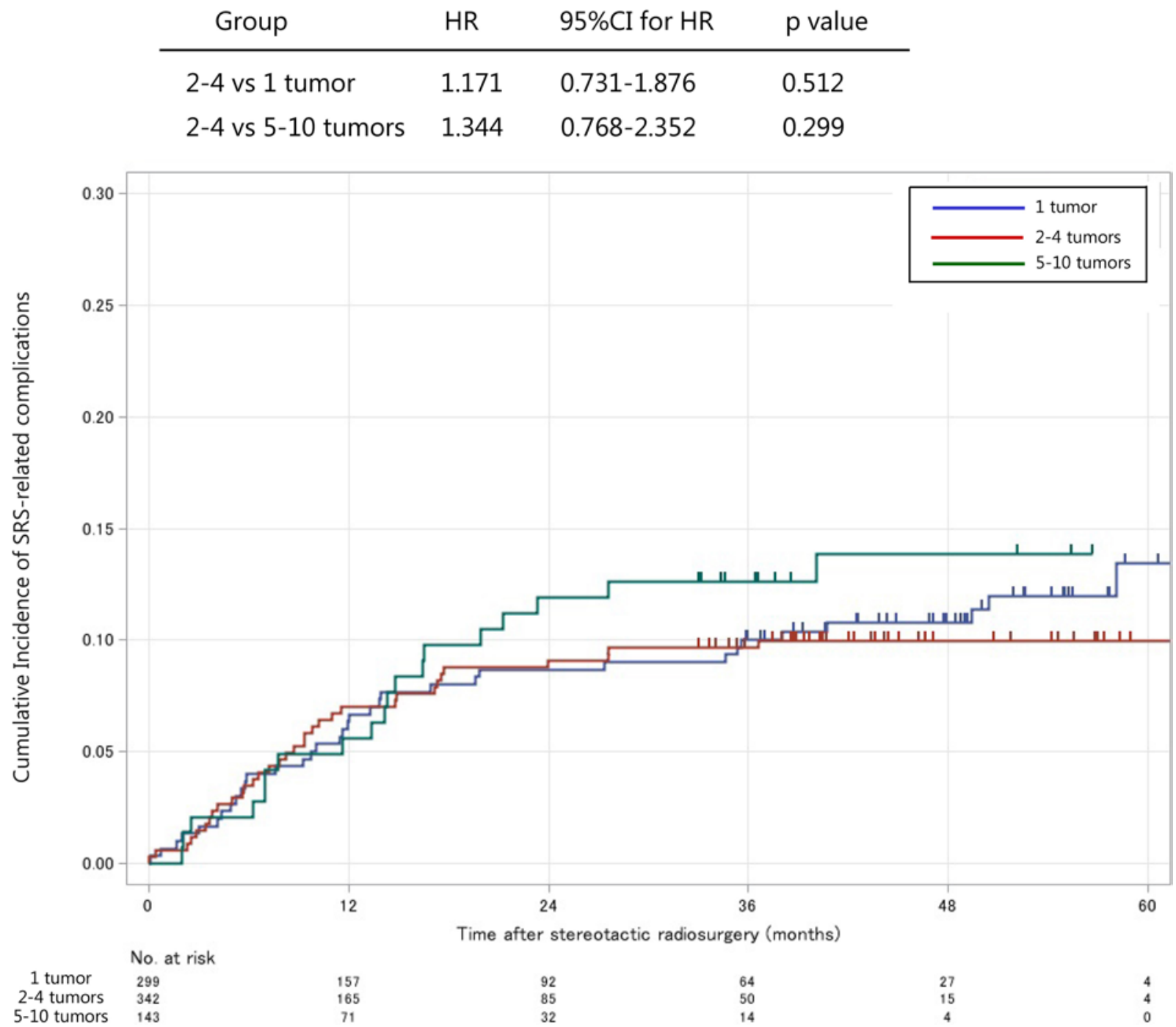

FIG. 3. Cumulative incidence rates of irradiation-related complications in patients after SRS in the 3 tumor-number groups, i.e., group A (1 tumor), group B (2-4 tumors), and group C (5-10 tumors).

multivariable (HR 1.165; 95\% CI 0.371-3.656; $\mathrm{p}=0.794)$ analysis detected a significant difference in SRS-related complication incidence rates between the 2 patient groups (i.e., those given a molecular targeting agent $[\mathrm{s}]$ alone versus those who were given another or no medication).

Follow-up MRI revealed leukoencephalopathy in 6 $(0.8 \%)$ of the 784 patients. In 5 of these 6 patients, the leukoencephalopathy had developed after salvage WBRT. A decline in the MMSE score during follow-up was noted in $2(33 \%)$ of the 6 patients.

The rates of NCF preservation 60 months after SRS were at least $85.7 \%$ in this cohort. The cumulative incidence rates of decreased MMSE scores are shown for the 3 tumor-number groups in Fig. 4. Differences among the 3 groups did not reach statistical significance. According to the multivariable analysis results, no clinical factors correlated with decreased NCF.

Many patients were managed by physicians other than us (such as clinicians who were providing them hospice care). As such, follow-up MMSE score data for some of our patients were not available. However, the statistical findings obtained are similar to previously reported results that were determined by applying analyses of missing data. ${ }^{22}$

\section{Discussion}

Based on this subset analysis of a group of patients with NSCLC exclusively (i.e., a more homogeneous patient group than that in our previous study), the noninferiority hypothesis of SRS alone as initial treatment for 5-10 BMs compared with that for 2-4 BMs, in terms of both overall survival and the majority of the secondary endpoints we examined, was clearly reconfirmed. Although these results do not differ fundamentally from those of our previous study, ${ }^{23}$ this subset analysis strengthens the aforementioned noninferiority hypothesis. The majority of published prospective/retrospective studies, even those that examined large numbers of patients who underwent SRS for BM, included highly heterogeneous patient char- 
TABLE 4. Clinical factors that affected posttreatment complications

\begin{tabular}{llll}
\hline \multicolumn{1}{c}{ Parameter } & & & $\begin{array}{c}p \\
\text { Value }\end{array}$ \\
\hline Group & HR & $95 \% \mathrm{Cl}$ & \\
\hline B vs A & 1.216 & $0.759-1.946$ & 0.416 \\
\hline C vs B & 1.251 & $0.704-2.222$ & 0.446 \\
\hline Sex, female vs male & 0.68 & $0.449-1.028$ & 0.067 \\
\hline Age, $\geq 65$ vs $<65$ yrs & 0.471 & $0.309-0.719$ & 0.001 \\
\hline KPS score, $\geq 80$ vs $<80$ & 0.607 & $0.300-1.228$ & 0.165 \\
\hline $\begin{array}{l}\text { Max diameter of largest tumor, }<1.6 \\
\text { vs } \geq 1.6 \text { cm }\end{array}$ & 1.345 & $0.534-3.387$ & 0.530 \\
\hline $\begin{array}{l}\text { Total tumor volume, }<1.9 \text { vs } \geq 1.9 \mathrm{~cm}^{3} \\
\text { Neurological symptoms, yes vs no }\end{array}$ & 1.201 & $0.480-3.001$ & 0.696 \\
\hline $\begin{array}{l}\text { Extracranial disease, not controlled } \\
\text { vs controlled }\end{array}$ & 0.817 & $0.516-1.293$ & 0.388 \\
\hline
\end{tabular}

acteristics (i.e., a range of primary cancers), although the most common primary site was the lung. The radiosensitivity and clinical responsiveness of BMs to treatment are generally regarded to depend on the oncological features of the primary malignancy. Therefore, in our view, it was reasonable to perform this subset study to focus solely on NSCLC.

Although WBRT has long been the gold standard among treatments for multiple BMs, the validity of using SRS alone to treat multiple BMs has been reconsidered recently. ${ }^{6,13,15}$ Furthermore, recent remarkable advancements in molecular targeted therapy have paved the way for updating therapeutic strategies for patients with lung cancer with BMs. Doherty et al. ${ }^{6}$ recently described treating BMs from epidermal growth factor receptor (EGFR)/anaplastic lymphoma kinase-positive NSCLC initially with WBRT (30 Gy in 10 fractions or 20 Gy in 5 fractions) plus a tyrosine kinase inhibitor (TKI) (120 patients), SRS (margin dose 15-21 Gy) plus a TKI (37 patients), or only a TKI (27 patients). The respective MSTs in their study were 21.6, 23.9 , and 22.6 months, showing no significant differences among their 3 groups. However, WBRT should not be the initial treatment for patients in whom prolonged survival can be expected because of the associated NCF decline. Therefore, a combination of SRS and a TKI is regarded as the most reasonable treatment strategy currently available for patients with NSCLC harboring multiple BMs. Magnuson et al. ${ }^{13}$ recently conducted a multicenter study of patients with EGFR mutation-positive NSCLC who

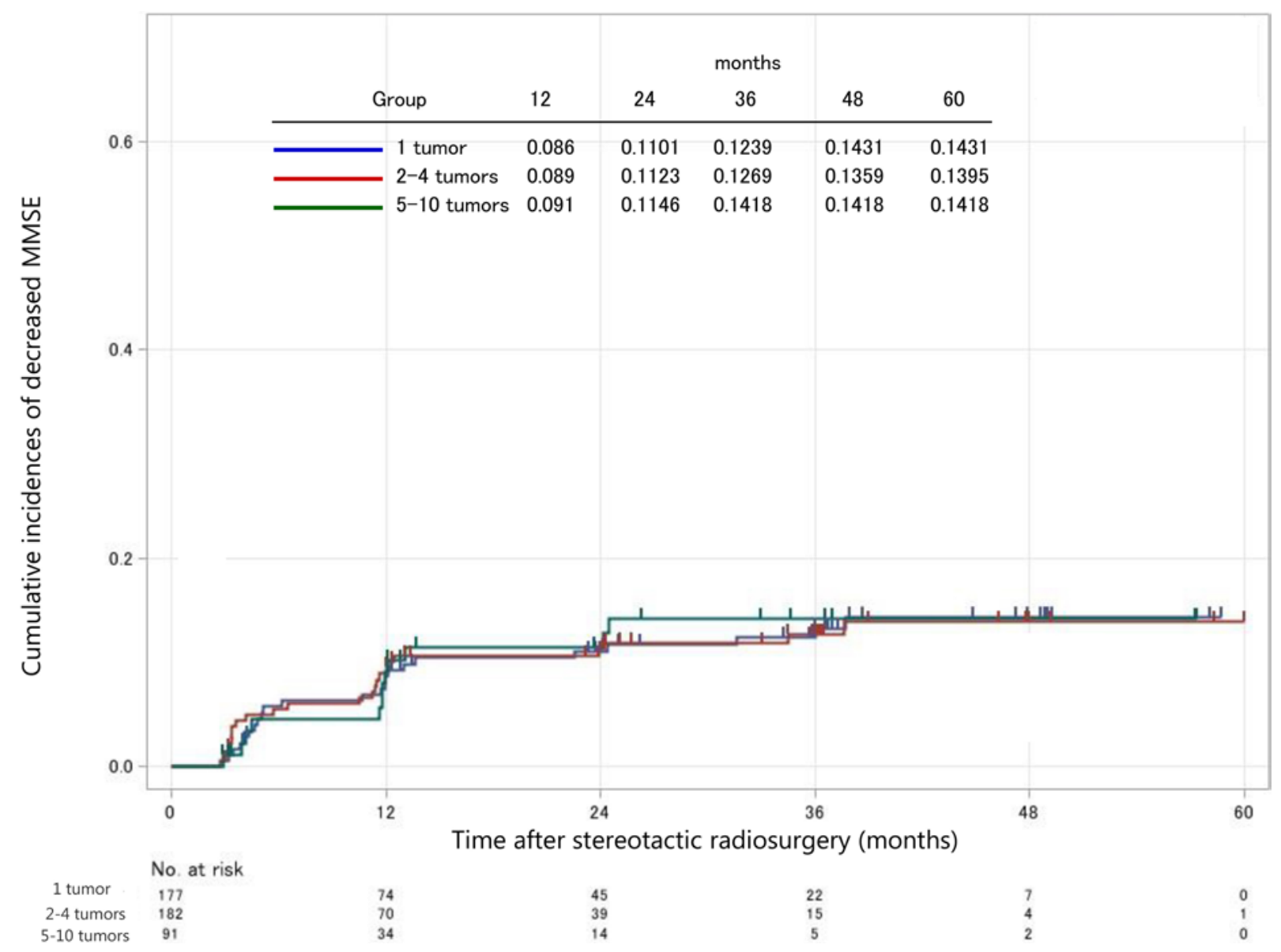

FIG. 4. Cumulative incidence rates of decreased MMSE scores after SRS for the 3 tumor-number groups, i.e., group A (1 tumor), group B (2-4 tumors), and group C (5-10 tumors). 
developed BMs. Their investigation yielded MSTs of 46 months with SRS, 30 months with WBRT, and 25 months with an EGFR-TKI regimen $(\mathrm{p}<0.001)$.

A major weakness of our study is that the JLGK0901 database did not include information regarding molecular subtypes. During the 2009-2012 patient enrollment period, only gefitinib and erlotinib were available in Japan. As noted already, patients who received molecular targeted agent therapy with or without a chemotherapeutic agent(s) survived significantly longer than those given either chemotherapy alone or no anticancer pharmacological agents.

In addition, immunotherapy that targets programmed cell death 1 (PD-1) or programmed cell death ligand 1 (PD-L1) recently became a standard treatment option. However, our database does not include patients given any of these therapies. Although immunotherapy for BMs that result from NSCLC is reportedly effective, ${ }^{7,9}$ the efficacy and risks associated with concurrent use of immune checkpoint inhibitors and SRS for BMs have not been well characterized yet. Chen et al. ${ }^{4}$ reported that the use of radiotherapy including SRS with concurrently administered immune checkpoint inhibitors might not raise the incidence of adverse events. Colaco et al..$^{5}$ suggested, however, that the rate of radiation necrosis after SRS for BMs might be increased in patients who undergo immunotherapy. Going forward, we anticipate that immunotherapy will play an increasingly important role in treating lung tumors in patients either with or without BMs. Thus, examining the efficacy and risks of combining SRS with anti-PD-1/PDL1 therapy is warranted.

SRS plus WBRT resulted in significantly diminished learning and memory functions, as measured by the Hopkins Verbal Learning Test-Revised (HVLT-R) total recall 4 months after treatment compared with that in the group given SRS only. ${ }^{2}$ Recent investigations of post-WBRT changes in NCF using the HVLT-R have been carried out for less than 2 years. ${ }^{2,20} \mathrm{~A}$ randomized trial found that the rates of HVLT-R total recall decline (52\%) and delayed recognition (22\%) were worse in the SRS-plus-WBRT group than in the SRS-only group (24\% and 6\%, respectively) 4 months after treatment. ${ }^{2}$ Although the MMSE battery used in our study recently received criticism because its sensitivity was lower than that of the HVLT-R and other batteries, our study has confirmed relatively low incidence rates of long-term (i.e., 4- to 5-year post-SRS) irradiation effects on the NCF of patients with lung cancer and BMs.

In our study, SRS-related complications were detected in $88(11 \%)$ patients during a median post-SRS period of 10.1 months, and this incidence rate was lower than or essentially equal to those obtained in other studies. ${ }^{12,19,21}$

Leukoencephalopathy was detected on follow-up MRI in $6(0.8 \%)$ of the 784 patients. We were not able to grade this complication because our protocol did not include a leukoencephalopathy-grading system. This complication was detected in 5 of 6 patients after salvage WBRT but in only 1 patient after SRS alone. Monaco et al $1{ }^{14}$ reported that leukoencephalopathy developed in only 1 of their 31 patients who underwent SRS alone, according to the final MRI studies. The risk of this complication after SRS alone seems to be very low.

The most significant criticism of SRS alone is the higher incidence of new distant BMs compared with that after SRS plus WBRT. Although WBRT is widely regarded as being able to prevent microscopic metastases, new BMs were reportedly discovered in $45 \%$ of the patients 6 months after WBRT. ${ }^{3}$ A study by Aoyama et al. ${ }^{1}$ found a $40 \%$ cumulative incidence of new lesion appearance after SRS plus WBRT. Our study found a $60 \%$ cumulative incidence of new lesion appearance 12 months after Gamma Knife SRS (group C), not significantly different than the rate in group B. These differences in rates were considered to be acceptable considering the difference in long-term risks of NCF deterioration between SRS alone and SRS plus WBRT. Gamma Knife SRS can be repeated if a new lesion were to appear, whereas WBRT can be performed only once. Because the optimal candidates for WBRT might be patients with leptomeningeal dissemination, using Gamma Knife SRS and reserving WBRT for patients with post-SRS dissemination provides a major clinical advantage.

Our long-term follow-up results show that $20 \%$ of the patients with BMs, all of whom met the inclusion criteria of the JLGK0901 multiinstitutional prospective study, survived for more than 3 years. Therefore, long-term preservation of NCF is clearly an essential element in selecting therapy for patients with BMs.

\section{Conclusions}

This subset analysis of patients with NSCLC reconfirmed the noninferiority of SRS alone in treating patients with 5-10 BMs compared with that in patients with 2-4 BMs in terms of both overall survival and secondary endpoints. Most notable is that neither the incidence of post-SRS complications nor NCF preservation differed significantly between groups B and C. These findings, in our view, strengthen the already-reported noninferiority hypothesis of SRS alone for patients with 5-10, compared with 2-4, BMs.

\section{Acknowledgments}

We express our thanks to all investigators involved in the JLGK study group who are not included here as coauthors. We also appreciate the efforts of Ms. Yukiko Hanawa (Tokyo Gamma Unit Center, Tsukiji Neurological Clinic) for her meticulous work in managing the database, and we thank Bierta E. Barfod (Katsuta Hospital Mito Gamma House) for assistance with English-language editing.

\section{References}

1. Aoyama H, Shirato H, Tago M, Nakagawa K, Toyoda T, Hatano K, et al: Stereotactic radiosurgery plus whole-brain radiation therapy vs stereotactic radiosurgery alone for treatment of brain metastases: a randomized controlled trial. JAMA 295:2483-2491, 2006

2. Chang EL, Wefel JS, Hess KR, Allen PK, Lang FF, Kornguth DG, et al: Neurocognition in patients with brain metastases treated with radiosurgery or radiosurgery plus whole-brain irradiation: a randomised controlled trial. Lancet Oncol 10:1037-1044, 2009

3. Chao ST, Barnett GH, Vogelbaum MA, Angelov L, Weil RJ, Neyman G, et al: Salvage stereotactic radiosurgery effectively treats recurrences from whole-brain radiation therapy. Cancer 113:2198-2204, 2008

4. Chen L, Douglass J, Kleinberg L, Ye X, Marciscano AE, 
Forde PM, et al: Concurrent immune checkpoint inhibitors and stereotactic radiosurgery for brain metastases in nonsmall cell lung cancer, melanoma, and renal cell carcinoma. Int J Radiat Oncol Biol Phys 100:916-925, 2018

5. Colaco RJ, Martin P, Kluger HM, Yu JB, Chiang VL: Does immunotherapy increase the rate of radiation necrosis after radiosurgical treatment of brain metastases? J Neurosurg 125:17-23, 2016

6. Doherty MK, Korpanty GJ, Tomasini P, Alizadeh M, Jao $\mathrm{K}$, Labbé C, et al: Treatment options for patients with brain metastases from EGFR/ALK-driven lung cancer. Radiother Oncol 123:195-202, 2017

7. Dudnik E, Yust-Katz S, Nechushtan H, Goldstein DA, Zer A, Flex D, et al: Intracranial response to nivolumab in NSCLC patients with untreated or progressing CNS metastases. Lung Cancer 98:114-117, 2016

8. Fine JP, Gray RJ: A proportional hazards model for the subdistribution of a competing risk. J Am Stat Assoc 94:496509, 1999

9. Gauvain C, Vauléon E, Chouaid C, Lerhun E, Jabot L, Scherpereel A, et al: Intracerebral efficacy and tolerance of nivolumab in non-small-cell lung cancer patients with brain metastases. Lung Cancer 116:62-66, 2018

10. Gooley TA, Leisenring W, Crowley J, Storer BE: Estimation of failure probabilities in the presence of competing risks: new representations of old estimators. Stat Med 18:695-706, 1999

11. Karnofsky D, Abelmann W, Craver L, Burchenal J: The use of nitrogen mustards in the palliative treatment of cancer. Cancer 1:634-656, 1948

12. Kohutek ZA, Yamada Y, Chan TA, Brennan CW, Tabar V, Gutin PH, et al: Long-term risk of radionecrosis and imaging changes after stereotactic radiosurgery for brain metastases. J Neurooncol 125:149-156, 2015

13. Magnuson WJ, Lester-Coll NH, Wu AJ, Yang TJ, Lockney NA, Gerber NK, et al: Management of brain metastases in tyrosine kinase inhibitor-naïve epidermal growth factor receptor-mutant non-small-cell lung cancer: a retrospective multi-institutional analysis. J Clin Oncol 35:1070-1077, 2017

14. Monaco EA III, Faraji AH, Berkowitz O, Parry PV, Hadelsberg U, Kano H, et al: Leukoencephalopathy after wholebrain radiation therapy plus radiosurgery versus radiosurgery alone for metastatic lung cancer. Cancer 119:226-232, 2013

15. Nabors LB, Portnow J, Ammirati M, Brem H, Brown P, Butowski N, et al: Central nervous system cancers, version 2.2014. Featured updates to the NCCN Guidelines. J Natl Compr Canc Netw 12:1517-1523, 2014

16. National Cancer Institute: Common Terminology Criteria for Adverse Events v3.0 (CTCAE). Bethesda, MD: National Institutes of Health, 2006 (https://ctep.cancer.gov/ protocoldevelopment/electronic_applications/docs/ctcaev3. pdf) [Accessed July 24, 2018]

17. Serizawa T, Hirai T, Nagano O, Higuchi Y, Matsuda S, Ono J, et al: Gamma Knife surgery for 1-10 brain metastases without prophylactic whole-brain radiation therapy: analysis of cases meeting the Japanese prospective multi-institute study (JLGK0901) inclusion criteria. J Neurooncol 98:163-167, 2010

18. Serizawa T, Yamamoto M, Sato Y, Higuchi Y, Nagano O, Kawabe T, et al: Gamma Knife surgery as sole treatment for multiple brain metastases: 2-center retrospective review of
1508 cases meeting the inclusion criteria of the JLGK0901 multi-institutional prospective study. J Neurosurg 113 Suppl:48-52, 2010

19. Sneed PK, Mendez J, Vemer-van den Hoek JG, Seymour ZA, Ma L, Molinaro AM, et al: Adverse radiation effect after stereotactic radiosurgery for brain metastases: incidence, time course, and risk factors. J Neurosurg 123:373-386, 2015

20. Sun A, Bae K, Gore EM, Movsas B, Wong SJ, Meyers CA, et al: Phase III trial of prophylactic cranial irradiation compared with observation in patients with locally advanced nonsmall-cell lung cancer: neurocognitive and quality-of-life analysis. J Clin Oncol 29:279-286, 2011

21. Yamamoto M, Kawabe T, Higuchi Y, Sato Y, Nariai T, Barfod BE, et al: Delayed complications in patients surviving at least 3 years after stereotactic radiosurgery for brain metastases. Int J Radiat Oncol Biol Phys 85:53-60, 2013

22. Yamamoto M, Serizawa T, Higuchi Y, Sato Y, Kawagishi J, Yamanaka K, et al: A multi-institutional prospective observational study of stereotactic radiosurgery for patients with multiple brain metastases (JLGK0901 study update): irradiation-related complications and long-term maintenance of Mini-Mental State Examination scores. Int J Radiat Oncol Biol Phys 99:31-40, 2017

23. Yamamoto M, Serizawa T, Shuto T, Akabane A, Higuchi Y, Kawagishi J, et al: Stereotactic radiosurgery for patients with multiple brain metastases (JLGK0901): a multi-institutional prospective observational study. Lancet Oncol 15:387-395, 2014

\section{Disclosures}

The authors report no conflict of interest concerning the materials or methods used in this study or the findings specified in this paper. This study was conducted with financial support, amounting to 11 million Japanese yen, from the Japan Brain Foundation (nongovernmental organization).

\section{Author Contributions}

Conception and design: Shuto, Akabane, Yamamoto, Serizawa, Higuchi. Acquisition of data: Shuto, Akabane, Yamamoto, Serizawa, Higuchi, Sato, Kawagishi, Yamanaka, Jokura, Yomo, Nagano. Analysis and interpretation of data: Shuto, Akabane, Yamamoto, Serizawa, Sato. Drafting the article: Shuto, Akabane, Yamamoto, Serizawa. Critically revising the article: Shuto, Akabane. Reviewed submitted version of manuscript: all authors. Statistical analysis: Sato. Administrative/technical/material support: Shuto, Akabane, Yamamoto, Serizawa. Study supervision: Yamamoto, Serizawa, Aoyama.

\section{Supplemental Information \\ Previous Presentations}

This work was presented in oral form at the 19th Leksell Gamma Knife Society Meeting, held in Dubai, United Arab Emirates, on March 5, 2018.

\section{Correspondence}

Takashi Shuto: Yokohama Rosai Hospital, Kanagawa, Japan. shuto@yokohamah.johas.go.jp. 